\title{
RESEARCH
}

Open Access

\section{Correlation between 12a-hydroxylated bile acids and insulin secretion during glucose tolerance tests in rats fed a high-fat and high-sucrose diet}

Reika Yoshitsugu ${ }^{1}$, Keidai Kikuchi ${ }^{1}$, Shota Hori ${ }^{1}$, Hitoshi Iwaya ${ }^{1}$, Masahito Hagio ${ }^{1}$, Hidehisa Shimizu ${ }^{2}$, Tohru Hira ${ }^{1}$ and Satoshi Ishizuka ${ }^{1 *}$ (iD

\begin{abstract}
Background: Previously, we found a significant relationship in a rat study between energy intake and bile acid (BA) metabolism especially $12 \mathrm{a}$-hydroxylated $(12 \mathrm{aOH})$ BAs. The present study was designed to reveal relationships among BA metabolism, glucose tolerance, and cecal organic acids in rats fed a high-fat and high-sucrose diet (HFS) by using multivariate and multiple regression analyses in two types of glucose tolerance tests (GTTs).

Methods: Male WKAH/HkmSlc rats were fed with a control or a HFS for 13 weeks. Oral glucose tolerance test (OGTT) and intraperitoneal glucose tolerance test (IPGTT) were performed at week 9 and 11, respectively. BAs were analyzed by using ultra high-performance liquid chromatography-mass spectrometry. Organic acid concentrations in cecal contents were analyzed by using ultra high-performance liquid chromatography with post-column $\mathrm{pH}$ buffered electric conductivity method.

Results: A positive correlation of aortic $12 \mathrm{aOH}$ BA concentration was observed with energy intake and visceral adipose tissue weight. We found that an increase of $12 \mathrm{aOH}$ BAs in enterohepatic circulation, intestinal contents and feces in the HFS-fed rats compared to those in control rats regardless of no significant increase of total BA concentration in the feces in the test period. Fecal $12 \mathrm{aOH}$ BA concentration was positively correlated with maximal insulin level in OGTT and area under curve of insulin in IPGTT. There was a positive correlation between aortic $12 \mathrm{aOH}$ BAs concentration and changes in plasma glucose level in both OGT and IPGTT. In contrast, a decrease in the concentration of organic acids was observed in the cecal contents of the HFS-fed rats. Multiple linear regression analysis in the IPGT revealed that the concentrations of aortic $12 \mathrm{aOH}$ BA and cecal acetic acid were the predictors of insulin secretion. Moreover, there was a positive correlation between concentration of portal $12 \mathrm{aOH}$ BAs and change in insulin concentration of peripheral blood in the IPGTT.
\end{abstract}

Conclusion: The distribution analysis of BA compositions accompanied by GTTs revealed a close relationship between $12 \mathrm{aOH}$ BA metabolism and insulin secretion in GTs in rats.

Keywords: Energy intake, Enterohepatic circulation, Glucose tolerance, Multiple regression analysis

\footnotetext{
*Correspondence: zuka@chem.agr.hokudai.ac.jp

${ }^{1}$ Research Faculty of Agriculture, Hokkaido University, Kita-9, Nishi-9, Kita-ku,

Sapporo 060-8589, Japan

Full list of author information is available at the end of the article
}

(c) The Author(s). 2020 Open Access This article is distributed under the terms of the Creative Commons Attribution 4.0 International License (http://creativecommons.org/licenses/by/4.0/), which permits unrestricted use, distribution, and reproduction in any medium, provided you give appropriate credit to the original author(s) and the source, provide a link to the Creative Commons license, and indicate if changes were made. The Creative Commons Public Domain Dedication waiver (http://creativecommons.org/publicdomain/zero/1.0/) applies to the data made available in this article, unless otherwise stated. 


\section{Background}

Non-communicable diseases (NCDs) includes cardiovascular disease, diabetes, cancer and chronic respiratory diseases [1]. Such NCDs are leading cause of death worldwide and the prevalence of NCDs is being increased [2]. Some behavioral factors including harmful use of alcohol, smoking tobacco, physical inactivity and unhealthy diet participate in development of NCDs [1], which suggest that dietary intervention is available to prevent NCDs. One of the devastating disorders is type 2 diabetes mellitus (T2DM) which is characterized as insulin resistance [3]. Recently, association is reported between insulin resistance and bile acid (BA) metabolism, especially $12 \alpha$-hydroxylated $(12 \alpha \mathrm{OH})$ BAs in humans $[4,5]$.

BA is one of the products in cholesterol catabolism [6] and structure of BAs is based on steroid ring with some hydroxyl groups and a carboxyl group, indicating amphipathic nature of the molecules, which contributes to lipid absorption in the upper small intestine [7] via bile secretion from the liver. In other words, an increase in BA concentration in the gut reflects energy intake in the animal body. BAs are usually reused via uptake by ileal epithelial cells that express sodium-dependent BA transporter [8] in the process referred to as enterohepatic circulation [6]. Since absorbed BAs in enterocytes are released into portal blood and incorporated in hepatocytes, BA concentration is considered to be higher in portal blood than in systemic blood. Those suggest BAs as a marker of energy intake in the body especially for organs related with enterohepatic circulation. Such alteration of BAs in an excess energy retention also can be detected in feces and blood.

A high energy intake not only increases a risk of diabetes but also induces alteration of BA metabolism. We observed that an increase in fecal concentration of $12 \alpha \mathrm{OH}$ BAs is positively associated with glucose intolerance in rats fed a HF diet [9]. Such increase in the concentration of $12 \alpha \mathrm{OH}$ BAs in a high energy intake might be involved in some early events in development of NCDs including T2DM. A HF and high-sucrose (HFS) diet is an energy-dense diet that includes a large amount of sucrose and also is a model at an early phase of T2DM [3]. To understand association between precise BA metabolism and glucose, we investigated alteration of biochemical parameters that reflect glucose tolerance in rats fed a HFS diet to identify significant relationships among those in an early phase of diabetes.

\section{Materials and methods}

Animals

Wistar King A Hokkaido male rats (WKAH/HkmSlc) (3week-old, Japan SLC, Shizuoka, Japan NBRP Rat No: 0154), an inbred strain of Wistar rats, were housed in an air-conditioned room at $22 \pm 2{ }^{\circ} \mathrm{C}$ temperature with $55 \pm$ $5 \%$ humidity, and the light period was from 8:00 to 20 : 00 . The rats were housed individually in wire-bottomed cages and allowed ad libitum access to diet and water, except for the periods performing glucose tolerance tests (GTT). Body weight and food consumption were measured every 2 days. Food consumption was calculated with weight difference of a feeding tray before and after feeding. The rats were acclimatized with a control diet based on the AIN-93G formulation (Table 1) [10]. The acclimatized rats $(n=22)$ were then divided into two groups and fed either the control $(C ; n=10)$ or a HFS diet $(n=12)$ for 13 weeks. Tail vein blood was collected from the rats in every 2 week from week 1 to 11 during the test period after food deprivation for $16 \mathrm{~h}$ to measure fasting plasma glucose concentration. Also, the feces were collected every 2 week from week 2 to 12 and in week 13 for measuring fecal BA compositions. At the end of the test period, bile juice was collected through a polyvinyl catheter (SV-35; id $0.5 \mathrm{~mm}$, od $0.9 \mathrm{~mm}$; Natsume Seisakusyo, Tokyo, Japan) inserted into common bile-pancreatic duct under anesthesia with sodium pentobarbital (Somnopentyl, $50 \mathrm{mg} / \mathrm{kg}$ body weight, Kyoritsu Seiyaku Corporation, Tokyo, Japan). The bile collection was performed for $10 \mathrm{~min}$ and the bile flow was measured. Immediately after the bile collection, the blood was collected from portal vein and aorta abdominalis, respectively. An anticoagulant reagent (heparin, $50 \mathrm{U} / \mathrm{mL}$ blood, Nakarai Tesque, Inc., Kyoto, Japan) and a protease inhibitor (aprotinin, $500 \mathrm{KIU} / \mathrm{mL}$ blood, Fujifilm Wako Pure Chemical Corporation, Osaka, Japan) were added to

Table 1 Diet compositions

\begin{tabular}{|c|c|c|}
\hline & $\begin{array}{l}\mathrm{C} \\
\mathrm{g} / \mathrm{kg} \mathrm{a}\end{array}$ & HFS \\
\hline Dextrin $^{a}$ & 529.5 & - \\
\hline Casein $^{b}$ & 200.0 & 200.0 \\
\hline Sucrose ${ }^{c}$ & 100.0 & 399.5 \\
\hline Soybean oil ${ }^{d}$ & 70.0 & 70.0 \\
\hline Lard & - & 230.0 \\
\hline Cellulose $^{e}$ & 50.0 & 50.0 \\
\hline Mineral mixture ${ }^{f}$ & 35.0 & 35.0 \\
\hline Vitamin mixture ${ }^{g}$ & 10.0 & 10.0 \\
\hline Choline bitartrate $^{\mathrm{h}}$ & 2.5 & 2.5 \\
\hline L-Cystine ${ }^{h}$ & 3.0 & 3.0 \\
\hline $\begin{array}{l}\text { a TK-16 (Matsutani Ch } \\
\text { b NZMP Acid Casein ( } \\
\text { New Zealand) } \\
{ }^{c} \text { Nippon Beet Sugar } \\
\text { d J-Oil Mills, Inc., Toky } \\
\text { e Crystalline cellulose } \\
{ }^{f} \text { AIN-93G mineral mix } \\
{ }^{g} \text { AIN-93 vitamin mixt } \\
\text { h Fujifilm Wako Pure }\end{array}$ & $\begin{array}{l}\text { Ltd., Hyo } \\
\text { e Group } \\
\text { d., Tokyo } \\
\text { hi Kasei }\end{array}$ & \\
\hline
\end{tabular}


Table 2 Food intake, growth, organ weights and bile flow

\begin{tabular}{lll}
\hline & $\mathrm{C}$ & HFS \\
\hline Cumulative food intake & & \\
$(\mathrm{g})$ & $1446 \pm 30$ & $1225 \pm 17^{*}$ \\
$(\mathrm{kcal})$ & $5707 \pm 121$ & $6244 \pm 88^{*}$ \\
Final body weight (g) & $372.4 \pm 11.5$ & $417.3 \pm 8.7^{*}$ \\
Organ weight (g/100 g body weight) & & \\
Liver & $2.97 \pm 0.05$ & $3.19 \pm 0.04^{*}$ \\
Visceral fat & $7.68 \pm 0.22$ & $8.94 \pm 0.14^{*}$ \\
Mesenteric adipose tissue & $1.49 \pm 0.07$ & $1.85 \pm 0.05^{*}$ \\
Epididymal adipose tissue & $2.66 \pm 0.07$ & $2.76 \pm 0.09$ \\
Retroperitoneal adipose tissue & $2.67 \pm 0.12$ & $3.52 \pm 0.08^{*}$ \\
Perirenal adipose tissue & $0.85 \pm 0.03$ & $0.81 \pm 0.02$ \\
Bile flow ( $\mu$ L/min) & $12.9 \pm 0.33$ & $15.8 \pm 1.09^{*}$ \\
\hline * Significant difference from the values in C (Student's $t$-test, $P<0.05, n=10$
\end{tabular}
for $\mathrm{C}, n=12$ for HFS)

the collected blood and the plasma was separated. The rats were killed by exsanguination thereafter. The liver, visceral adipose tissues, and intestinal contents were collected and weighed.

\section{BA analysis}

The amount of each BA in the bile, intestinal contents (jejunum, ileum, and cecum), and feces were measured after extraction as previously reported $[9,11,12]$. The individual BA concentrations were measured with nordeoxycholic acid (23-nor-5 $\beta$-cholanic acid-3 $\alpha, 12 \alpha-$ diol) as an internal standard.

$12 \alpha \mathrm{OH}$ BAs measured in this experiment were as follows: cholic acid (CA; $5 \beta$-cholanic acid-3 $\alpha, 7 \alpha, 12 \alpha$-triol), deoxy-

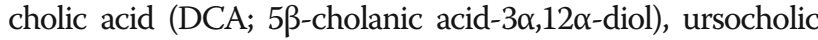
acid (UCA; $5 \beta$-cholanic acid-3 $\alpha, 7 \beta, 12 \alpha$-triol), taurocholic acid (TCA; $5 \beta$-cholanic acid-3 $\alpha, 7 \alpha, 12 \alpha-$ triol- $N$-(2-sulfoethyl)-amide), taurodeoxycholic acid (TDCA; $5 \alpha$-cholanic acid-3 $\alpha, 12 \alpha$-diol- $N$-(2-sulfoethyl)-amide,), glycocholic acid (GCA; $5 \beta$-cholanic acid-3 $\alpha, 7 \alpha, 12 \alpha$-triol- $N$-(carboxymethyl)-

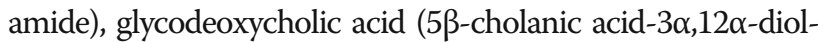
$N$-(carboxymethyl)-amide), 7-oxo-deoxycholic acid (7oDCA; $5 \beta$-cholanic acid-3 $\alpha, 12 \alpha$-diol-7-one), 12-oxo-lithocholic acid (12oLCA; $5 \beta$-cholanic acid-3 $\alpha$-ol-12-one), and $5 \beta$-cholanic acid-12 $\alpha$-ol-3-one $(3 o 12 \alpha)$.

Non-12 $\alpha \mathrm{OH}$ BAs measured in this experiment were as fol-

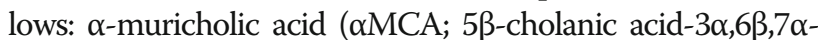
triol), $\beta$-muricholic acid ( $\beta$ MCA; $5 \beta$-cholanic acid-3 $\alpha, 6 \beta$, $7 \beta$-triol), $\omega$-muricholic acid ( $\omega$ MCA; $5 \beta$-cholanic acid-3 $3,6 \alpha$, $7 \beta$-triol), chenodeoxycholic acid (CDCA; $5 \beta$-cholanic acid-

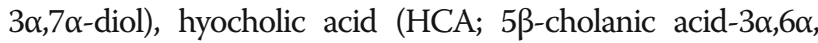
$7 \alpha$-triol), hyodeoxycholic acid (HDCA; $5 \beta$-cholanic acid- $3 \alpha$, $6 \alpha$-diol), ursodeoxycholic acid (UDCA; $5 \beta$-cholanic acid-3 $\alpha$, $7 \beta$-diol), lithocholic acid (LCA; $5 \beta$-cholanic acid-3 $\alpha$-ol), tauro- $\alpha$-muricholic acid (T $\alpha$ MCA; $5 \beta$-cholanic acid- $3 \alpha, 6 \beta$, $7 \alpha$-triol- $N$-(2-sulfoethyl)-amide), tauro- $\beta$-muricholic acid

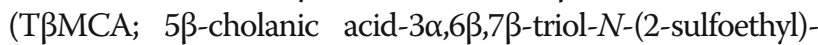

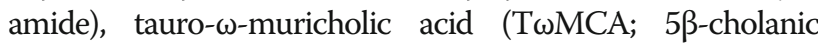
acid-3 $\alpha, 6 \alpha, 7 \beta$-triol- $N$-(2-sulfoethyl)-amide), $\quad$ taurochenodeoxycholic acid (TCDCA; $5 \beta$-cholanic acid-3 $\alpha, 7 \alpha$-diol- $N$ -

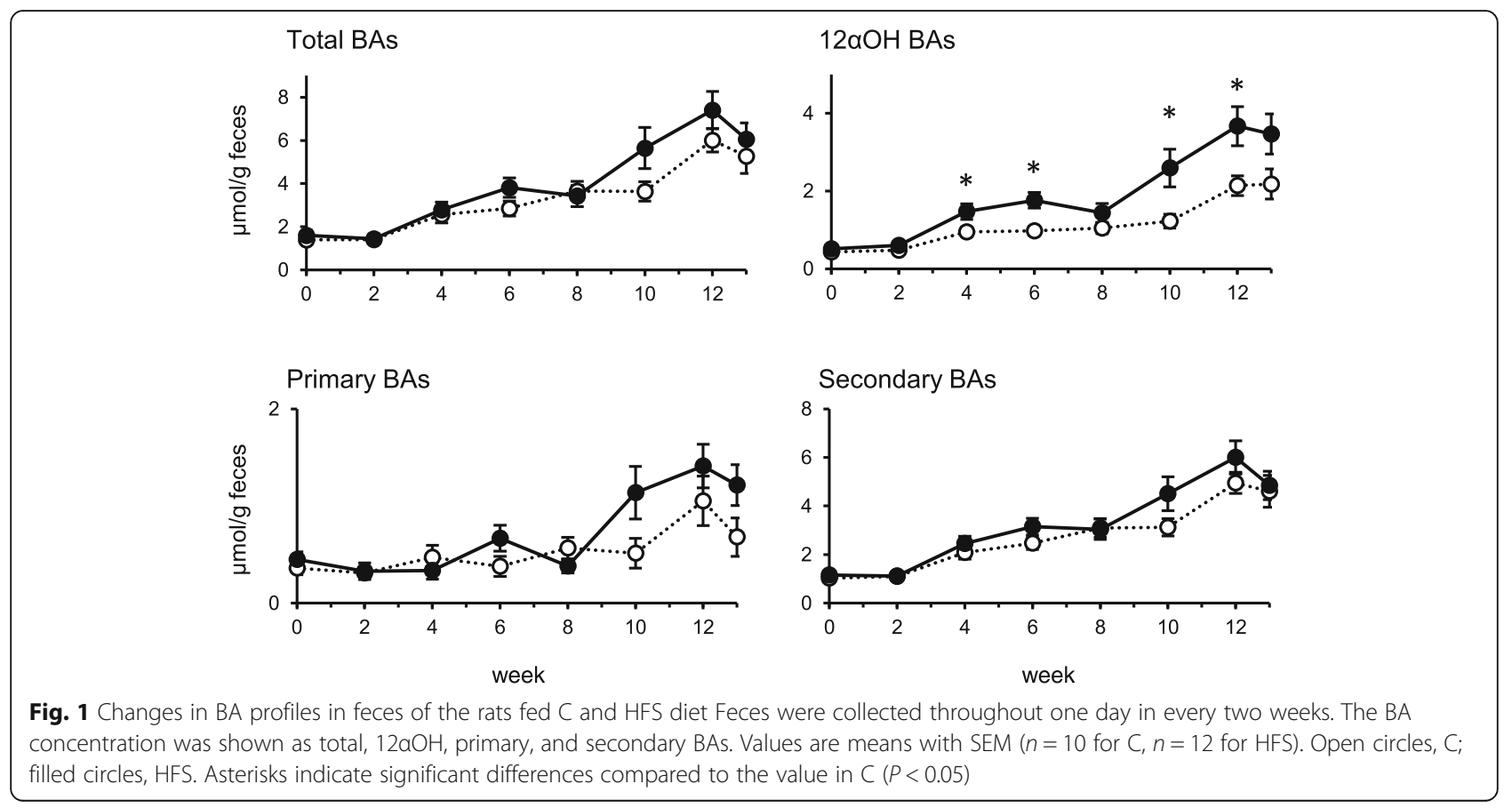


(2-sulfoethyl)-amide), taurohyodeoxycholic acid (5ß-cholanic acid-3 $\alpha, 6 \alpha$-diol- $N$-(2-sulfoethyl)-amide), taurolithocholic acid (5 $\beta$-cholanic acid-3 $\alpha$-ol- $N$-(2-sulfoethyl)-amide), glycocheno-

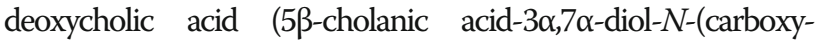
methyl)-amide), glycohyodeoxycholic acid (5 $\beta$-cholanic acid- $3 \alpha$, $6 \alpha$-diol- $N$-(carboxymethyl)-amide), glycoursodeoxycholic acid

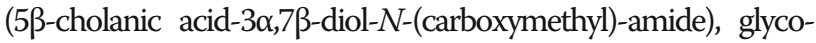

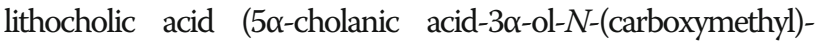
amide), and 7-oxo-lithocholic acid (7oLCA; 5ß-cholanic acid-3 $\alpha$-ol-7-one).
All BAs, with the exception of UCA, were purchased from Steraloids, Inc. (Newport, RI, USA); UCA was obtained from Toronto Research Chemicals (Toronto, Ontario, Canada).

\section{Oral and Intraperitoneal glucose tolerance tests}

We performed two types of GTT with oral or intraperitoneal administrations [13] at week 9 and 11, respectively. IPGTT was performed to observe direct response to glucose without influence of its absorption in the intestine. The rats were fasted for $16 \mathrm{~h}$ beforehand and fasting blood

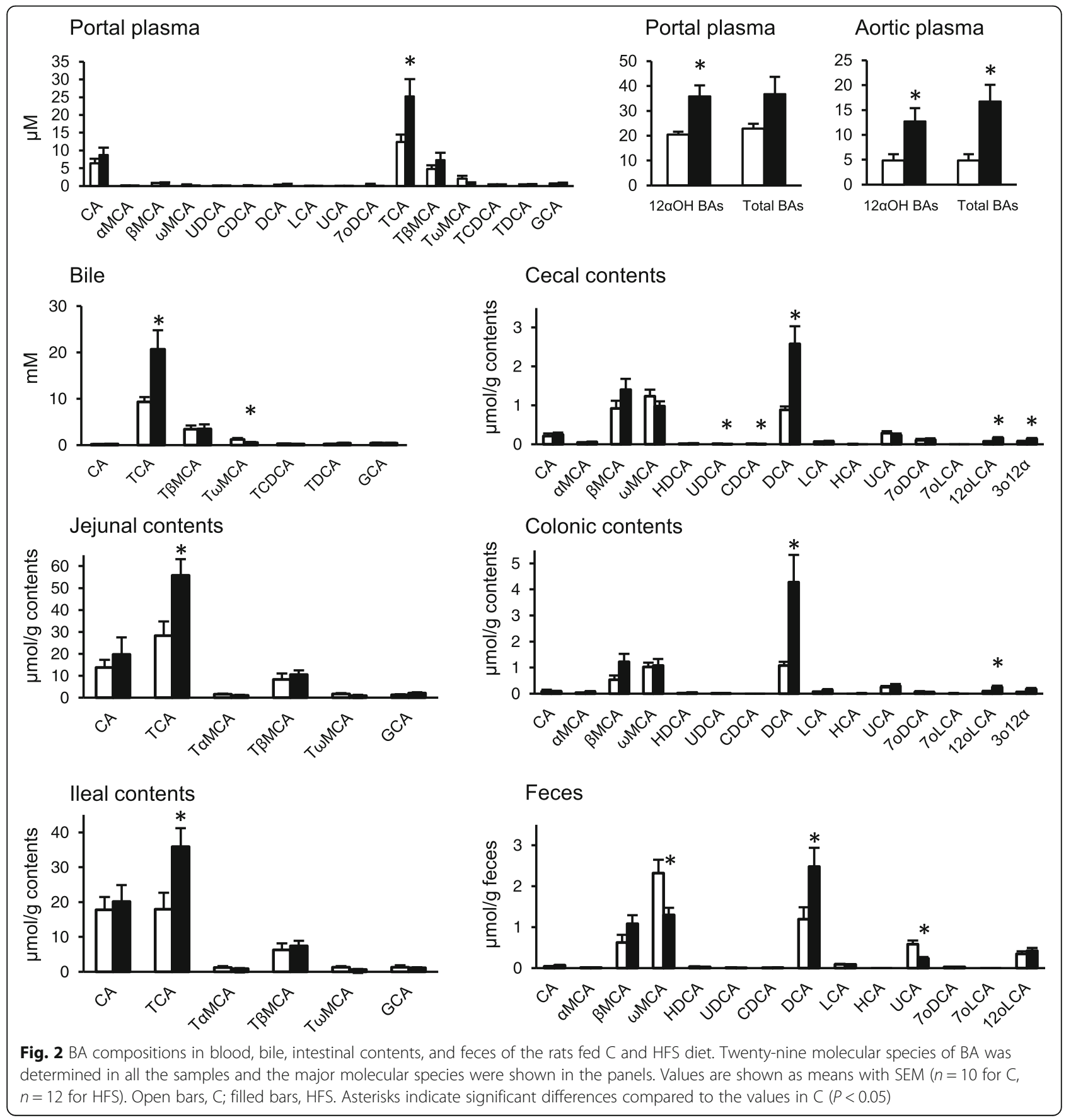



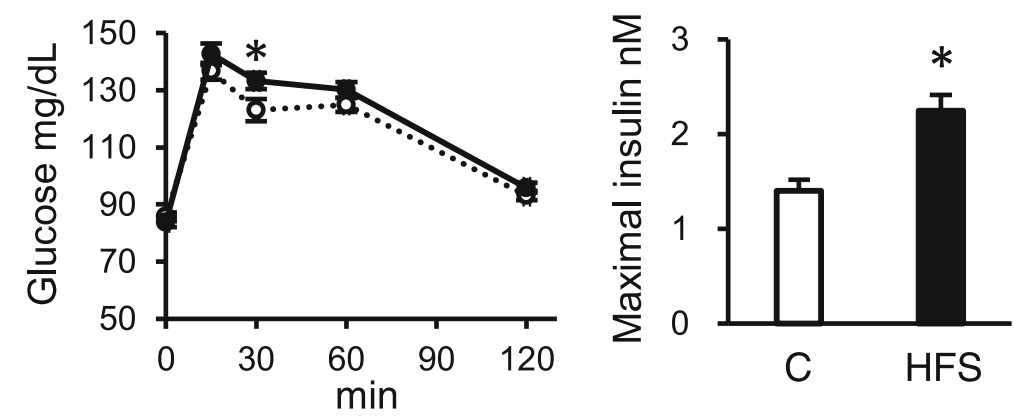

Fig. 3 Changes in glucose and insulin concentrations in the blood plasma from tail vein of the rats fed control and HFS diet in oral glucose tolerance test (OGTT) at week 9. After collection of tail vein blood in fasting condition ( $16 \mathrm{~h}$ food deprivation) for time 0 , a glucose solution was orally administered ( $2 \mathrm{~g} / \mathrm{kg}$ rats) and tail vein blood was collected at the indicated time points to measure glucose. Maximal insulin concentration in blood was determined at 15 min postadministration of glucose. Values are shown as means with SEM ( $n=10$ for $C, n=12$ for HFS). Open circles, C; filled circles, HFS. Asterisks indicate significant differences compared to the values in $C(P<0.05)$

was collected from the tail vein. A glucose solution was administered orally (OGTT; $2 \mathrm{~g} / \mathrm{kg}$ ) at week 9 or intraperitoneally (IPGTT; $1 \mathrm{~g} / \mathrm{kg}$ ) at week 11 . At the indicated time points after glucose injection, blood samples were collected from the tail vein into tubes containing heparin and aprotinin as mentioned above. The plasma was separated by centrifugation and stored at $-80^{\circ} \mathrm{C}$ until analysis. The plasma glucose and insulin concentrations were analyzed using Glucose CII-test kit (Wako) and Rat Insulin ELISA kit (AKRIN-010 T) (Fujifilm Wako Shibayagi Coporation, Shibukawa, Japan), respectively. Area under curve (AUC) in plasma glucose and insulin was calculated with the trapezoidal rule in each GTT. For calculation of $\triangle \mathrm{AUC}$, the glucose or insulin concentration at the starting point was set to 0 . Then, the changes in blood glucose or insulin concentration was plotted in every time point and $\triangle \mathrm{AUC}$ was from those values.

\section{Organic acid analysis in cecal contents}

Organic acids in the cecal contents were measured by using HPLC (Shimadzu Corporation, Kyoto, Japan) with crotonic acid (Fujifilm Wako Pure Chemical Corportion) as an internal standard [14]. Briefly, the cecal contents were homogenized and neutralized with sodium hydroxide. The hydrophobic substances in the supernatant were removed by chloroform, and the aqueous phase was passed through a membrane filter (cellulose acetate, $0.20 \mu \mathrm{m}$ pore size; DISMIC13cp; Toyo Roshi Kaisha, Ltd., Tokyo, Japan). The samples were analyzed by HPLC (Shimadzu Corporation) equipped with a solvent delivery system (SCL10AVP; Shimadzu Corporation), a double ionexchange column (Shim-Pack SCR-102H, $8 \mathrm{~mm} \times 300$ $\mathrm{mm}$; Shimadzu), and an electro-conductivity detector (CDD-6A; Shimadzu Corporation). The mobile phase was $5 \mathrm{mM}$ of $p$-toluenesulfonic acid, and the detection solution was $5 \mathrm{mM}$ of $p$-toluenesulfonic acid containing $100 \mu \mathrm{M}$ of EDTA and $20 \mathrm{mM}$ of Bis-Tris.

\section{Statistical analyses}

The significance of differences between $\mathrm{C}$ and HFS groups was determined using Student's $t$-test. Multivariate correlation analysis was performed in each GTT test. Multiple linear regression was performed to determine predictors in various parameters to insulin $\triangle \mathrm{AUC}$ during the IPGTT. Pearson's method was used to evaluate simple regression. A $P$ value $<0.05$ was considered to be significant. JMP version 14.0.0 (SAS Institute Inc., Cary, NC, USA) was used for the statistical analyses.

\section{Results}

There was a decrease in diet consumption in the HFSfed rats (Table 2) presumably due to energy density. On the other hand, total energy intake was significantly higher in the HFS-fed rats than in C. The body weight in the HFS-fed rats was greater than that in C accompanied by an increased weight of visceral adipose tissues along with the energy intake. There was a significant increase in relative liver weight in the HFSfed rats than in $\mathrm{C}$-fed rats $(P<0.05)$. Consumption of the HFS diet enhanced basal secretion of the bile juice from the liver.

We measured fecal BA concentration during the experiment (Fig. 1). No apparent difference was found in the concentrations of total, primary and secondary BAs. Actual fecal BAs excretion was almost the same because of no significant difference in fecal weight between the groups (data not shown). A significantly higher concentration of $12 \alpha \mathrm{OH}$ BAs in feces was observed in the HFS-

Table 3 Parameters in OGT in rats fed C or HFS at week 9

\begin{tabular}{lll}
\hline & $\mathrm{C}$ & HFS \\
\hline Fasting glucose $\mathrm{mg} / \mathrm{dL}$ & $85.7 \pm 1.57$ & $83.8 \pm 1.67$ \\
Glucose AUC mg/dL·h & $232.8 \pm 3.5$ & $241.6 \pm 3.1$ \\
Glucose $\Delta$ AUC $\mathrm{mg} / \mathrm{dL} \cdot \mathrm{h}$ & $60.5 \pm 4.4$ & $74.0 \pm 3.9^{*}$ \\
\hline
\end{tabular}

* Significant difference from the values in C (Student's $t$-test, $P<0.05, n=10$ for $\mathrm{C}, n=12$ for HFS) 

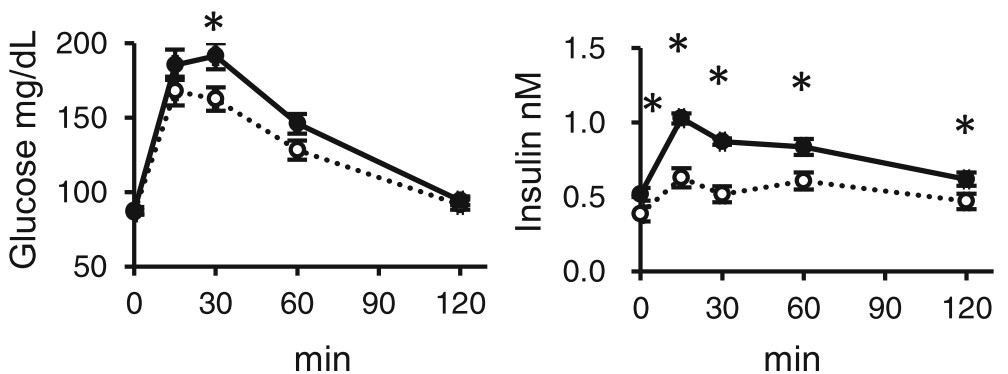

Fig. 4 Changes in glucose and insulin concentrations in the blood plasma from tail vein of the rats fed C and HFS diet in intraperitoneal glucose tolerance test (IPGTT) at week 11. After collection of tail vein blood in fasting condition (16 h food deprivation) for time 0 , a glucose solution was intraperitoneally injected ( $1 \mathrm{~g} / \mathrm{kg}$ rats) and tail vein blood was collected at the indicated time points to measure glucose and insulin concentrations. Values are shown as means with SEM ( $n=10$ for $C, n=12$ for HFS). Open circles, C; filled circles, HFS. Asterisks indicate significant differences compared to the value in $C(P<0.05)$

fed rats at week 4, 6, 10 and 12. In the BA composition at the end of the experiment (Fig. 2), TCA was found to be the major component of BAs in bile juice, small intestine (jejunal and ileal contents) and portal blood especially in the HFS-fed rats. The concentrations of $12 \alpha \mathrm{OH}$ BAs and total BAs in aorta blood were lower than that of portal blood. The BA composition in large intestinal contents (cecum and colon) and feces was almost comparable and a high concentration of DCA was found in the large intestinal contents and feces preferentially in the HFS-fed rats. In C, sum of the MCAs (mainly BMCA and $\omega \mathrm{MCA}$ ) was much abundant rather than DCA in the large intestine and feces. In contrast, DCA turned to be the major BA molecule in the HFS-fed rats.

In the OGTT at weeks 9, the blood glucose was significantly higher at $30 \mathrm{~min}$ post administration (Fig. 3) and a significant increase was observed in the glucose $\triangle$ AUC in the HFS-fed rats (Table 3 ). The maximum insulin concentration was higher in the HFS-fed rats than in C-fed rats at $15 \mathrm{~min}$ after oral administration of glucose (Fig. 3). Multiple correlation analysis in the parameters in OGTT revealed significant positive correlations of aorta $12 \alpha \mathrm{OH}$ BA concentration with energy intake, visceral fat, and glucose $\triangle \mathrm{AUC}$ (Additional file 1: Table S1). Fecal $12 \alpha \mathrm{OH}$ BA concentration was also correlated with the maximal insulin level.

In the IPGTT study performed at week 11 (Fig. 4), there was a significant increase of the peak glucose concentration in the HFS-fed rats in response to the glucose injection compared to that in C-fed rats. Also, significant increases were observed in insulin concentration throughout the IPGTT in the HFS-fed rats. Such changes were confirmed in the insulin AUC and the $\triangle$ AUC (Table 4). There was an increase in fasting leptin concentration in the HFS-fed rats (Table 4). No difference was observed in the glucose-related parameters, such as fasting glucose, glucose AUC, glucose $\triangle A U C$ as well as HOMA-IR between the dietary groups.
We observed significant decreases in the concentration of succinic acid, acetic acid, propionic acid as well as nbutyric acid (Fig. 5). Multivariate correlation analysis in IPGTT at week 11 (Additional file 2: Table S2) showed that aorta $12 \alpha \mathrm{OH}$ BAs at week 13 was positively correlated with energy intake, liver weight, visceral fat, glucose $\triangle$ AUC. Fecal $12 \alpha \mathrm{OH}$ BAs correlated with insulin $\triangle A U C$, and negatively associated with cecal butyrate level. Multiple linear regression analysis in the IPGTT (Table 5) revealed that changes in aortic $12 \alpha \mathrm{OH}$ BA concentration and cecal acetic acid were predictors of insulin $\triangle A U C$ in the IPGTT. We also found a significant positive correlation between concentration of $12 \alpha \mathrm{OH}$ BAs in portal plasma and insulin AUC in the IPGTT (Fig. 6).

\section{Discussion}

HF feeding in rodents is a diet-induced obese model and displays a variety of disorders such as obesity, insulin resistance and/or dyslipidemia [3]. There is a few paper that describes $12 \alpha \mathrm{OH}$ BA metabolism in these experiments. However, an increase in $12 \alpha \mathrm{OH}$ BA concentration was observed in tissues related with enterohepatic circulation in rats [9] and there is an association

Table 4 Parameters in IPGTT in rats fed C or HFS at week 11

\begin{tabular}{|c|c|c|}
\hline & C & HFS \\
\hline Fasting glucose $\mathrm{mg} / \mathrm{dL}$ & $84.1 \pm 2.05$ & $89.1 \pm 1.99$ \\
\hline Glucose AUC mg/dL·h & $255.5 \pm 9.56$ & $285.5 \pm 11.7$ \\
\hline Glucose $\triangle A \cup C$ mg/dL·h & $82.9 \pm 10.3$ & $111.0 \pm 11.6$ \\
\hline Fasting insulin $\mathrm{nM}$ & $0.38 \pm 0.05$ & $0.53 \pm 0.04^{*}$ \\
\hline Insulin AUC nM•h & $1.09 \pm 0.10$ & $1.60 \pm 0.06^{*}$ \\
\hline Insulin $\triangle A \cup C \mathrm{nM} \cdot \mathrm{h}$ & $0.32 \pm 0.06$ & $0.55 \pm 0.06^{*}$ \\
\hline Relative HOMA-IR & $1.00 \pm 0.13$ & $1.38 \pm 0.13$ \\
\hline Fasting leptin $\mu \mathrm{g} / \mathrm{mL}$ & $120.4 \pm 21.7$ & $238.3 \pm 20.1^{*}$ \\
\hline
\end{tabular}

* Significant difference from the values in C (Student's $t$-test, $P<0.05, n=10$ for $\mathrm{C}, n=12$ for HFS) 


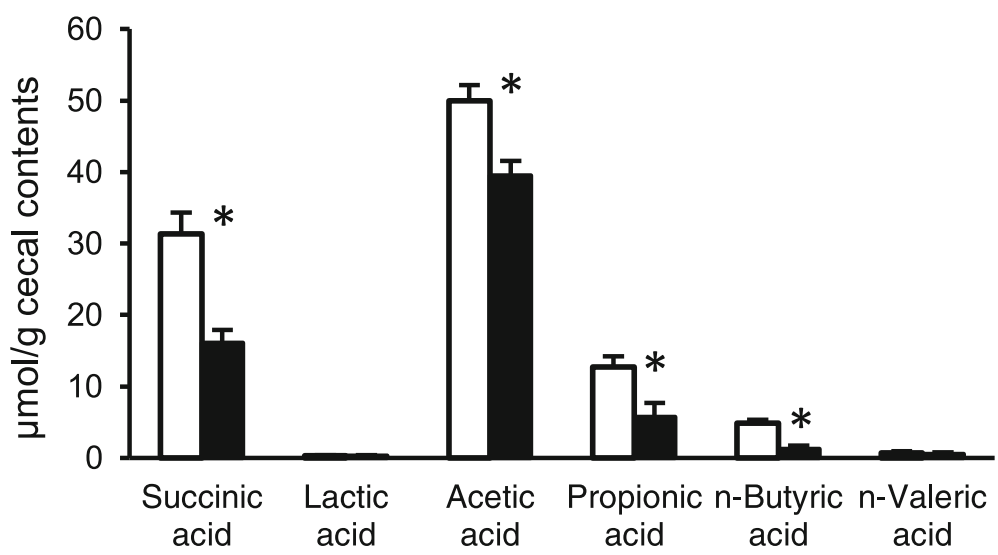

Fig. 5 Organic acid concentrations in the cecal contents of the rats fed C and HFS diet. Values are shown as means with SEM $(n=10$ for $C, n=$ 12 for HFS). Open circles, C; filled circles, HFS. Asterisks indicate significant differences compared to the value in $C(P<0.05)$

between plasma $12 \alpha \mathrm{OH}$ BA concentration and insulin resistance in humans [4]. These observations suggest that $12 \alpha \mathrm{OH}$ BA metabolism is involved in alteration of glucose metabolism in these conditions. The present study revealed precise BA metabolism in HFS-fed rats such as significant increase in fecal $12 \alpha \mathrm{OH}$ BA concentration in HFS-fed rats regardless of no difference in fecal total BA concentration between the groups. An increase in fecal total BAs might be found if we have much longer experimental period as we observed in our previous experiment [9]. On the other hand, enormous increase was observed in $12 \alpha \mathrm{OH}$ BA concentration, especially in bile, small intestinal contents, and portal plasma in the HFS-fed rats, which suggests a selective recycle of $12 \alpha \mathrm{OH}$ BAs in enterohepatic circulation in the HFS-fed condition. In contrast, non- $12 \alpha \mathrm{OH}$ BA seemed to be easily excreted in feces of HFS-fed rats. This study also revealed a significant correlation between portal $12 \alpha \mathrm{OH}$ BA concentration and insulin secretion in IPGTT (Fig. 6). These results suggest a close association between enterohepatic $12 \alpha \mathrm{OH}$ BAs circulation and glucose metabolism in rats.

As shown in Fig. 2, HFS diet induced selective increase in the concentration of TCA, one of the $12 \alpha \mathrm{OH}$ BAs, in the ileal contents and portal blood. TCA is possible to induce insulin secretion from $\beta$ cells in pancreas via Gpbar1/TGR5 [15-17]. These studies suggest a direct

Table 5 Predictors of changes in insulin concentration of the rats in IPGTT

\begin{tabular}{lll}
\hline Variables & $\beta$ & $P$-value \\
\hline Aortic 12aOH BAs & 0.46 & 0.0078 \\
Cecal acetic acid & -0.58 & 0.0015 \\
\hline
\end{tabular}

Multiple linear regression analysis was performed to determine various parameters to insulin $\triangle$ AUC during the IPGTT $(n=22)$. The changes in aortic $12 \mathrm{aOH} B A s$ and cecal acetic acid concentration explained $52 \%$ of the total variance in the model as predictors of insulin $\triangle \mathrm{AUC} . R^{2}=0.568$, adjusted $R^{2}=$ $0.521, d f=2, F=11.85, P=0.0005$, Durbin-Watson $=1.71$ role of TCA in insulin secretion. Since an increase of insulin secretion was observed during OGTT in portal plasma of the rats fed a HFS diet in our previous study [13], it is possible that TCA enhances insulin secretion from $\beta$ cells. Additionally, TCA induces glucagon-like peptide-1 (GLP-1) secretion via Gpbar1/TGR5 in STC-1 cells, an enteroendocrine cell line [18], suggesting promotion of insulin secretion via GLP-1 from enteroendocrine L-cells. Actually, there was a positive association between $12 \alpha \mathrm{OH}$ BAs and insulin secretion in the present study (Fig. 6). Although we did not determine insulin resistance directly, it is possible that the increase in insulin secretion in the HFS-fed rats was partially due to the increase in $12 \alpha \mathrm{OH}$ BA concentration in the enterohepatic circulation regardless of insulin sensitivity.

Acetic acid derived from dietary fiber and nondigestible saccharides by fermentation in large intestine can

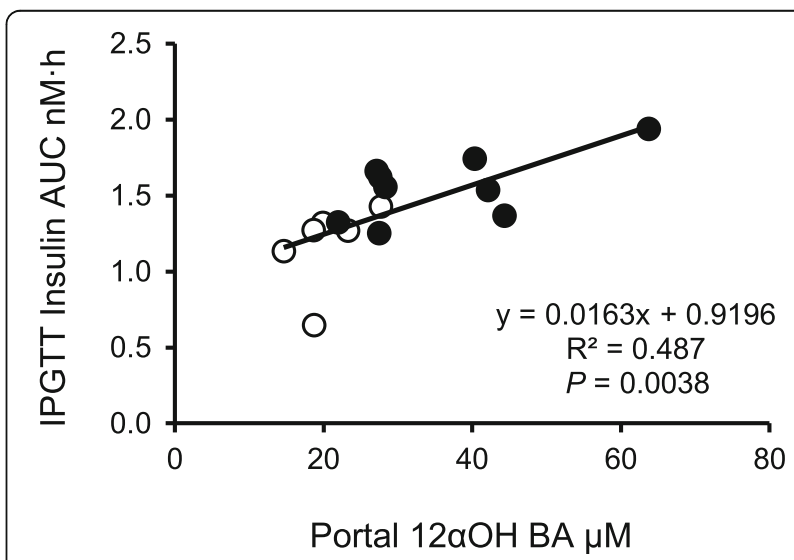

Fig. 6 Relationship between concentration of $12 \mathrm{aOH}$ BAs in portal plasma and insulin AUC in the IPGTT. Each plot shows individual values. Open circles, C; filled circles, HFS. The insulin AUC was calculated from the data shown in Fig. 4. The portal 12aOH BAs concentration was calculated the data as shown in Fig. 2. Pearson's method was used to evaluate simple regression 
activate $5^{\prime}$-AMP-activated protein kinase (AMPK) [1921]. Considering AMPK activation contributes to insulin sensitivity [22], those observations suggest that the decrease of acetic acid concentration in the cecal contents of the rats fed with the HFS diet plays a role in insulin tolerance. In the present study, acetic acid in the cecal contents was the predictor of the insulin $\triangle \mathrm{AUC}$ together with $12 \alpha \mathrm{OH}$ BA in aortic plasma by the multiple correlation analysis (Table 5), suggesting that increase in $12 \alpha \mathrm{OH}$ BA and reduction in cecal acetic acid concentration contribute to enhancement of insulin secretion.

Several studies show that BA depletion influences glucose homeostasis by BA sequestration (BAS) or abrogation of enzymes responsible for BA production, such as Cyp7a1 and Cyp8b1 as follows. BAS ameliorates glucose homeostasis in $o b / o b$ mice [23], $d b / d b$ mice [24], fatty liver Shionogi mice [25], and also in humans [26]. Those observations suggest that depletion of BAs from intestinal luminal contents improves glucose homeostasis in both humans and mice. In mice, abrogation of Cyp7a1, the rate-limiting enzyme in BA synthesis [27], improves glucose metabolism in mice fed a HF/high-cholesterol diet. Additionally, a dietary supplementation of CA at $0.03 \%$ deteriorates glucose metabolism in the Cyp7a1 KO mice. Another report shows that a retarded glucose clearance in mice fed high-cholesterol diet is normalized by abrogation of Cyp8b1, the enzyme responsible for production of $12 \alpha \mathrm{OH}$ BAs [28].

Although the present experiment did not allow an increase in the concentration of total BAs in feces of the rats fed HFS diet during the experimental period (Fig. 1), we could detect a significant increase of $12 \alpha \mathrm{OH}$ BA concentration in the feces. Such increase in the proportion of $12 \alpha \mathrm{OH}$ BAs may be induced by steroidogenic acute regulatory protein (StAR) that transfers cholesterol to mitochondria to produce non- $12 \alpha \mathrm{OH}$ BAs [29]. The expression of StAR is attenuated in a HF-fed mice and over expression of StAR improves insulin resistance induced by a HF diet [29]. An upregulation of Cyp8b1 in the liver might be another option to enhance the proportion of $12 \alpha \mathrm{OH}$ BAs because Cyp8b1 is constitutively expressed in liver [30]. In comparison with our previous experiment in rats fed a HF diet [9], substitution of carbohydrate source from starch to sucrose may not influence the BA metabolism by the lard supplementation in the diet because almost no difference was observed in the BA metabolism of the rats fed either HF [9] or HFS diet (Fig. 2).

Another interesting observation in comparison between the present and our previous studies was a difference in relative liver weights between the HFS-fed rats in the present study and the HF-fed rats in our previous study [9]. As expected, an increase was observed in relative weights of visceral adipose tissues in the rats fed either the HFS or HF diet. On the contrary, the HFS diet increased relative liver weight as compared with that in control (Table 2) whereas the HF diet rather decreased the relative liver weight in our previous study [9]. The difference in the relative liver weight between HFS and HF is considered to depend on carbohydrate source. Obviously, the HFS diet contains nearly $40 \%$ of sucrose in the diet that includes $20 \%$ of fructose in weight basis in the diet. It is suggested that fructose in the HFS diet seems to be a source of steatosis probably via fatty acid synthesis [31]. The increase in BA concentration in the enterohepatic circulation of a HF-fed rats might have a role in the incorporation of fructose as well as glucose in hepatocytes, resulting in fatty acid synthesis. Such enlargement of liver size might be in relation with hepatic lipid accumulation that deteriorates glucose homeostasis.

\section{Conclusions}

Both $12 \alpha \mathrm{OH}$ BAs and cecal acetic acid were predictors for insulin secretion in multiple regression analysis. Additionally, there was a significant positive correlation between portal $12 \alpha \mathrm{OH}$ BA concentration and insulin secretion in the IPGTT. These results revealed an association between $12 \alpha \mathrm{OH}$ BA metabolism and insulin secretion in GTTs in rats. Modulation of $12 \alpha \mathrm{OH}$ BAs and organic acid in the gut might be available to normalize glucose homeostasis in a high-energy consumption.

\section{Supplementary information}

Supplementary information accompanies this paper at https://doi.org/10. 1186/s12944-020-1193-2.

Additional file 1: Table S1. Correlation between each pair of variables in growth, OGT, and $12 \mathrm{aOH}$ BAs.

Additional file 2: Table S2. Multiple correlations between each pair of variables in growth, IPGTT, $12 \mathrm{aOH}$ BAs, and cecal organic acids.

\section{Abbreviations}

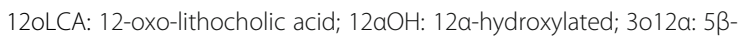
cholanic acid-12a-ol-3-one; 7oDCA: 7-oxo-deoxycholic acid; 7oLCA: 7-oxolithocholic acid; AMPK: AMP-activated protein kinase; BA: Bile acid; C: Control; CA: Cholic acid; CDCA: Chenodeoxycholic acid; DCA: Deoxycholic acid; FGF: Fibroblast growth factor; GCA: Glycocholic acid; GLP-1: Glucagon-like peptide-1; GT: Glucose tolerance test; HCA: Hyocholic acid; HDCA: Hyodeoxycholic acid; HF: High-fat; HFS: High-fat and high-sucrose; IPGT: Intraperitoneal glucose tolerance test; LCA: Lithocholic acid; MCA: Muricholic acid; NCD: Noncommunicable disease; OGTT: Oral glucose tolerance test; StAR: Steroidogenic acute regulatory protein; T2DM: Type-2 diabetes mellitus; TCA: Taurocholic acid; TCDCA: Taurochenodeoxycholic acid; TDCA: Taurodeoxycholic acid; TaMCA: Tauro-a-muricholic acid;

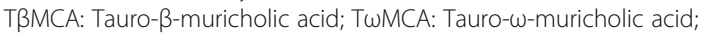
UCA: Ursocholic acid; UDCA: Ursodeoxycholic acid

\section{Acknowledgements}

Not applicable.

\section{Authors' contributions}

$\mathrm{SI}$ designed and supervised the implementation of this study; RY, KK, HI, and $\mathrm{MH}$ performed experiments; $\mathrm{RY}, \mathrm{SI}, \mathrm{HS}, \mathrm{TH}$, and $\mathrm{SH}$ analyzed data and wrote the paper; All the authors read and approved the final manuscript. 


\section{Funding}

This work was supported by JSPS KAKENHI Grant Numbers 16 K14917, $19 H 2900$ and JST COI Grant Number JPMJCE1301. The authors declare no conflicts of interest.

\section{Availability of data and materials}

The datasets analyzed during the current study are included in the manuscript.

\section{Ethics approval and consent participate}

The present study was approved by the Institutional Animal Care and Use Committee of National Corporation, Hokkaido University (approval number: 14-0026 and 17-0119), and all animals were maintained in accordance with the Hokkaido University Manual for Implementing Animal Experimentation.

\section{Consent for publication}

Not applicable.

\section{Competing interests}

The authors declare that they have no competing interests.

\section{Author details}

${ }^{1}$ Research Faculty of Agriculture, Hokkaido University, Kita-9, Nishi-9, Kita-ku, Sapporo 060-8589, Japan. ${ }^{2}$ Institute of Life and Environmental Science, Academic Assembly, Shimane University, Matsue 690-8504, Japan.

Received: 14 November 2019 Accepted: 10 January 2020

Published online: 15 January 2020

\section{References}

1. Alwan A. Global status report on noncommunicable diseases 2010. World Health Organization; 2011. https://www.who.int/nmh/publications/ncd_ report2010/en/

2. Riley L, Cowan M. Noncommunicable diseases country profiles. World Health Organization; 2018. https://www.who.int/nmh/publications/ncdprofiles-2018/en/.

3. Kleinert M, Clemmensen C, Hofmann SM, Moore MC, Renner S, Woods SC, Huypens $P$, Beckers J, de Angelis MH, Schürmann A, Bakhti M, Klingenspor M, Heiman M, Cherrington AD, Ristow M, Lickert H, Wolf E, Havel PJ, Müller TD, Tschöp MH. Animal models of obesity and diabetes mellitus. Nat Rev Endocrinol. 2018;14:140-62.

4. Haeusler RA, Astiarraga B, Camastra S, Accili D, Ferrannini E. Human insulin resistance is associated with increased plasma levels of 12a-hydroxylated bile acids. Diabetes. 2013:62:4184-91.

5. Wewalka M, Patti ME, Barbato C, Houten SM, Goldfine AB. Fasting serum taurine-conjugated bile acids are elevated in type 2 diabetes and do not change with intensification of insulin. J Clin Endocrinol Metab. 2014;99: 1442-51.

6. Zhou H, Hylemon PB. Bile acids are nutrient signaling hormones. Steroids. 2014:86:62-8

7. Murphy C, Parini P, Wang J, Björkhem I, Eggertsen G, Gåfvels M. Cholic acid as key regulator of cholesterol synthesis, intestinal absorption and hepatic storage in mice. Biochim Biophys Acta. 1735;2005:167-75.

8. Oelkers P, Kirby LC, Heubi JE, Dawson PA. Primary bile acid malabsorption caused by mutations in the ileal sodium-dependent bile acid transporter gene (SLC10A2). J Clin Invest. 1997;99:1880-7.

9. Yoshitsugu R, Kikuchi K, Iwaya H, Fujii N, Hori S, Lee DG, Ishizuka S Alteration of bile acid metabolism by a high-fat diet is associated with plasma transaminase activities and glucose intolerance in rats. J Nutr Sci Vitaminol. 2019;65:45-51

10. Reeves PG, Nielsen FH, Fahey GC Jr. AlN-93 purified diets for laboratory rodents: final report of the American Institute of Nutrition ad hoc writing committee on the reformulation of the AIN-76A rodent diet. J Nutr. 1993; 123:1939-51.

11. Hagio M, Matsumoto M, Fukushima M, Hara H, Ishizuka S. Improved analysis of bile acids in tissues and intestinal contents of rats using LC/ESI-MS. J Lipid Res. 2009:50:173-80.

12. Hagio M, Matsumoto M, Ishizuka S. Bile acid analysis in various biological samples using ultra performance liquid chromatography/electrospray ionization-mass spectrometry (UPLC/ESI-MS). Methods Mol Biol. 2011;708: 119-29.
13. Hira T, Suto R, Kishimoto $Y$, Kanahori $S$, Hara H. Resistant maltodextrin or fructooligosaccharides promotes GLP-1 production in male rats fed a highfat and high-sucrose diet, and partially reduces energy intake and adiposity. Eur J Nutr. 2018:57:965-79.

14. Hoshi S, Sakata T, Mikuni K, Hashimoto H, Kimura S. Galactosylsucrose and xylosylfructoside alter digestive tract size and concentrations of cecal organic acids in rats fed diets containing cholesterol and cholic acid. J Nutr. 1994;124:52-60.

15. Kumar DP, Rajagopal S, Mahavadi S, Mirshahi F, Grider JR, Murthy KS, Sanyal AJ. Activation of transmembrane bile acid receptor TGR5 stimulates insulin secretion in pancreatic $\beta$ cells. Biochem Biophys Res Commun. 2012;427: 600-5.

16. Shapiro H, Kolodziejczyk AA, Halstuch D, Elinav E. Bile acids in glucose metabolism in health and disease. J Exp Med. 2018;215:383-96.

17. Maczewsky J, Kaiser J, Gresch A, Gerst F, Düfer M, Krippeit-Drews P, Drews G. TGR5 activation promotes stimulus-secretion coupling of pancreatic $\beta$ cells via a PKA-dependent pathway. Diabetes. 2019;68:324-36.

18. Katsuma S, Hirasawa A, Tsujimoto G. Bile acids promote glucagon-like peptide-1 secretion through TGR5 in a murine enteroendocrine cell line STC-1. Biochem Biophys Res Commun. 2005;329:386-90.

19. Sakakibara S, Yamauchi T, Oshima Y, Tsukamoto Y, Kadowaki T. Acetic acid activates hepatic AMPK and reduces hyperglycemia in diabetic KK-A(y) mice. Biochem Biophys Res Commun. 2006;344:597-604.

20. Kondo T, Kishi M, Fushimi T, Kaga T. Acetic acid upregulates the expression of genes for fatty acid oxidation enzymes in liver to suppress body fat accumulation. J Agric Food Chem. 2009:57:5982-6.

21. Petsiou El, Mitrou PI, Raptis SA, Dimitriadis GD. Effect and mechanisms of action of vinegar on glucose metabolism, lipid profile, and body weight. Nutr Rev. 2014;72:651-61.

22. Ruderman NB, Carling D, Prentki M, Cacicedo JM. AMPK, insulin resistance, and the metabolic syndrome. J Clin Invest. 2013:123:2764-72.

23. Solís N, Pizarro M, Quintero P, Arab JP, Riquelme A, Padilla O, Carrasco G, Pirola CJ, Sookoian S, Arrese M. Effects of bile acid sequestration on hepatic steatosis in obese mice. Ann Hepatol. 2013;13:105-12.

24. Yamakawa T, Ogihara K, Utsunomiya H, Muraoka T, Kadonosono K, Terauchi Y. Colestimide improves glycemic control via hepatic glucose production in db/db mice. Endocr J. 2014:61:425-36.

25. Tagawa H, Irie J, Itoh A, Kusumoto Y, Kato M, Kobayashi N, Tanaka K, Morinaga R, Fujita M, Nakajima Y, Morimoto K, Sugizaki T, Kawano Y, Yamada S, Kawai T, Watanabe M, Itoh $\mathrm{H}$. Bile acid binding resin improves hepatic insulin sensitivity by reducing cholesterol but not triglyceride levels in the liver Diabetes Res Clin Pract. 2015:109:85-94.

26. Marina AL, Utzschneider KM, Wright LA, Montgomery BK, Marcovina SM, Kahn SE. Colesevelam improves oral but not intravenous glucose tolerance by a mechanism independent of insulin sensitivity and $\beta$-cell function. Diabetes Care. 2012;35:1119-25.

27. Ferrell JM, Boehme S, Li F, Chiang JY. Cholesterol 7a-hydroxylase-deficient mice are protected from high-fat/high-cholesterol diet-induced metabolic disorders. J Lipid Res. 2016:57:1144-54.

28. Kaur A, Patankar JV, de Haan W, Ruddle P, Wijesekara N, Groen AK, Verchere CB, Singaraja RR, Hayden MR. Loss of Cyp8b1 improves glucose homeostasis by increasing GLP-1. Diabetes. 2015;64:1168-79.

29. Qiu Y, Sui X, Zhan Y, Xu C, Li X, Ning Y, Zhi X, Yin L. Steroidogenic acute regulatory protein (StAR) overexpression attenuates HFD-induced hepatic steatosis and insulin resistance. Biochim Biophys Acta Mol basis Dis. 1863; 2017:978-90.

30. Bertaggia E, Jensen KK, Castro-Perez J, Xu Y, Di Paolo G, Chan RB, Wang L, Haeusler RA. Cyp8b1 ablation prevents Western diet-induced weight gain and hepatic steatosis because of impaired fat absorption. Am J Physiol Endocrinol Metab. 2017;313:E121-33.

31. Ackerman Z, Oron-Herman M, Grozovski M, Rosenthal T, Pappo O, Link G, Sela BA. Fructose-induced fatty liver disease: hepatic effects of blood pressure and plasma triglyceride reduction. Hypertension. 2005;45:1012-8.

\section{Publisher's Note}

Springer Nature remains neutral with regard to jurisdictional claims in published maps and institutional affiliations. 